PROCEEDINGS OF THE

AMERICAN MATHEMATICAL SOCIETY

Volume 135, Number 9, September 2007, Pages 2949-2960

S 0002-9939(07)08838-7

Article electronically published on February 9, 2007

\title{
ON $L^{2}$ COHOMOLOGY OF ACH KÄHLER MANIFOLDS
}

\author{
XIAODONG WANG
}

(Communicated by Richard A. Wentworth)

\begin{abstract}
We discuss a class of complete Kähler manifolds which are asymptotically complex hyperbolic near infinity. The main result is a sharp vanishing theorem for the second $L^{2}$ cohomology of such manifolds under certain assumptions. The borderline case characterizes a Kähler-Einstein manifold constructed by Calabi.
\end{abstract}

\section{INTRODUCTION}

Thanks to the $A d S / C F T$ correspondence in string theory, the study of conformally compact Einstein manifolds has been very active and many new results have been obtained; see, e.g., Anderson [A], Witten and Yau [WY], and Wang W1, etc. From a mathematical point of view, it is very natural to also study manifolds whose asymptotic geometry is locally modeled on the other rank one symmetric spaces, namely the complex hyperbolic space $\mathbb{C} \mathbb{H}^{n}$, the quarternionic hyperbolic space $\mathbb{H} \mathbb{H}^{n}$ and the Cayley plane $\mathbb{O} \mathbb{H}^{n}$. Such manifolds, especially those which are asymptotic to the complex hyperbolic space, have been studied by a few authors, first Epstein and Melrose [EM] and more recently Biquard [Biq]. In this paper we focus on Kähler manifolds which are asymptotically complex hyperbolic (ACH) near infinity. We show that a number of results and methods in the study of conformally compact Einstein manifolds can be generalized to these manifolds. We try to obtain optimal results in the Kähler setting.

The paper is organized as follows. In Section 2, we discuss a noncompact Kähler -Einstein manifold constructed by Calabi. This is an interesting example on which we can produce explicitly an $L^{2}$-harmonic $(1,1)$-form and also determine the bottom of the spectrum of the Laplacian on functions. In Section 3 we prove some vanishing theorems on $L^{2}$-harmonic forms on a noncompact Kähler manifold asymptotic to the complex hyperbolic space. In Section 4, we discuss the borderline case and prove that this characterizes the Calabi example. In the last section we make a few remarks and raise some open questions.

\section{An example of Calabi}

Let $\pi: L \rightarrow M$ be a holomorphic line bundle over a compact manifold $M$ of complex dimension $n$. Suppose $L$ is endowed with a Hermitian metric such that

$$
\theta=2 \sqrt{-1} \partial \bar{\partial} \log |\sigma|^{2},
$$

Received by the editors January 17, 2006 and, in revised form, May 24, 2006.

2000 Mathematics Subject Classification. Primary 53C55; Secondary 58 J50.

(C)2007 American Mathematical Society 
where $\sigma$ is a holomorphic section, $\bar{\partial}$ is a Kähler form on $M$ and

$$
\operatorname{Ric}(\theta)=-\frac{n+1}{2} \theta .
$$

Then Calabi constructed the following Kähler-Einstein metric on the disc bundle $P=\{\sigma \in L|| \sigma \mid<1\}$ :

$$
\omega=\frac{1}{1-|\sigma|^{2}} \theta+\frac{2|\sigma|^{2}}{\left(1-|\sigma|^{2}\right)^{2}} \sqrt{-1} \partial \log |\sigma|^{2} \wedge \bar{\partial} \log |\sigma|^{2} .
$$

For the construction, see Calabi Cal. With the metric $\omega$ explicitly given, it is easy to verify that $\operatorname{Ric}(\omega)=-\frac{n+2}{2} \omega$. First we have

$$
\omega^{n+1}=\frac{2(n+1)|\sigma|^{2}}{\left(1-|\sigma|^{2}\right)^{n+2}} \theta^{n} \wedge \sqrt{-1} \partial \log |\sigma|^{2} \wedge \bar{\partial} \log |\sigma|^{2} .
$$

Therefore, by (2.1) and (2.2),

$$
\begin{aligned}
\operatorname{Ric}(\omega) & \\
= & \operatorname{Ric}(\theta)-\sqrt{-1} \partial \bar{\partial} \log |\sigma|^{2}+(n+2) \sqrt{-1} \partial \bar{\partial} \log \left(1-|\sigma|^{2}\right) \\
= & \operatorname{Ric}(\theta)+\left(n+1-\frac{n+2}{1-|\sigma|^{2}}\right) \sqrt{-1} \partial \bar{\partial} \log |\sigma|^{2} \\
& -\frac{(n+2)|\sigma|^{2}}{\left(1-|\sigma|^{2}\right)^{2}} \sqrt{-1} \partial \log |\sigma|^{2} \wedge \bar{\partial} \log |\sigma|^{2} \\
= & -\frac{n+2}{2\left(1-|\sigma|^{2}\right)} \theta-\frac{(n+2)|\sigma|^{2}}{\left(1-|\sigma|^{2}\right)^{2}} \sqrt{-1} \partial \log |\sigma|^{2} \wedge \bar{\partial} \log |\sigma|^{2} \\
= & -\frac{n+2}{2} \omega .
\end{aligned}
$$

Moreover if $(M, \theta)$ is complex hyperbolic, i.e., all the holomorphic sectional curvatures are -1 , then $(P, \omega)$ is also complex hyperbolic. This makes the Calabi construction geometrically transparent in this special case. Namely if $M$ is complex hyperbolic, we can represent it as $B^{n} / \Gamma$, where $B^{n}$ is the unit ball in $\mathbb{C}^{n}$ with the Bergmann metric and $\Gamma$ is a torsion cocompact lattice in $\operatorname{PSU}(1, n)$, the isometry group of $B^{n}$. Then $P$ is simply $B^{n+1} / \Gamma$, where $\Gamma$ is viewed as a discrete subgroup of $P S U(1, n+1)$ via the natural inclusion $P S U(1, n) \rightarrow P S U(1, n+1)$.

Consider the following real $(1,1)$-form on $P$ :

$$
\xi=f\left(|\sigma|^{2}\right)\left(\theta-\frac{2 n|\sigma|^{2}}{1-|\sigma|^{2}} \sqrt{-1} \partial \log |\sigma|^{2} \wedge \bar{\partial} \log |\sigma|^{2}\right)
$$

where $f(t)=(1-t)^{n}$. One easily verifies that $\Lambda \xi \omega^{n+1}=(n+1) \omega^{n} \wedge \xi=0$ and

$$
\begin{aligned}
\partial \xi & =f^{\prime} \partial|\sigma|^{2} \wedge \theta+\frac{2 n f|\sigma|^{2}}{1-|\sigma|^{2}} \sqrt{-1} \partial \log |\sigma|^{2} \wedge \partial \bar{\partial} \log |\sigma|^{2} \\
& =|\sigma|^{2}\left(f^{\prime}+\frac{n f}{1-|\sigma|^{2}}\right) \partial \log |\sigma|^{2} \wedge \theta \\
& =0 .
\end{aligned}
$$


Similarly $\bar{\partial} \xi=0$. By the Kähler identities $\xi$ is harmonic. By a simple calculation and (2.4), we have

$$
\begin{aligned}
\omega^{n-1} \wedge \xi^{2} & =-2(n+1)\left(1-|\sigma|^{2}\right)^{n}|\sigma|^{2} \theta^{n} \wedge \sqrt{-1} \partial \log |\sigma|^{2} \wedge \bar{\partial} \log |\sigma|^{2} \\
& =-\left(1-|\sigma|^{2}\right)^{2(n+1)} \omega^{n+1} .
\end{aligned}
$$

This implies

$$
|\xi|^{2}=n(n+1)\left(1-|\sigma|^{2}\right)^{2(n+1)} .
$$

By the formula (2.4) for the volume form, it is then easy to see that $\xi$ is $L^{2}$ integrable. In fact $u=|\xi|^{n /(n+1)}=c_{n}\left(1-|\sigma|^{2}\right)^{n} \in L^{2}$.

Proposition 2.1. We have $u \in L^{2}$ and $-\Delta u=n u$.

Proof. The first claim is obvious. Let $f=1-|\sigma|^{2}$. We compute

$$
\begin{aligned}
|\nabla f|^{2} & =2 \Lambda(\sqrt{-1} \partial u \wedge \bar{\partial} f) \\
& =2|\sigma|^{4} \Lambda\left(\sqrt{-1} \partial \log |\sigma|^{2} \wedge \bar{\partial} \log |\sigma|^{2}\right) \\
& =|\sigma|^{2}\left(1-|\sigma|^{2}\right)^{2} \\
& =(1-f) f^{2} \\
-\Delta f & =-2 \sqrt{-1} \Lambda \partial \bar{\partial} f \\
& =2|\sigma|^{2} \sqrt{-1} \Lambda\left(\partial \bar{\partial} \log |\sigma|^{2}+\partial \log |\sigma|^{2} \wedge \bar{\partial} \log |\sigma|^{2}\right) \\
& =\left(1-|\sigma|^{2}\right)\left(1+(n-1)|\sigma|^{2}\right) \\
& =n f-(n-1) f^{2} .
\end{aligned}
$$

By these two identities, we easily obtain

$$
-\Delta f^{n}=-n f^{n-1} \Delta f-n(n-1) f^{n-2}|\nabla f|^{2}=n f^{n} .
$$

The proposition states that $u$ is a positive $L^{2}$ eigenfunction with eigenvalue $n$. It is then easy to see that $n$ is in fact the bottom of the spectrum of $-\Delta$.

\section{VANISHING THEOREMS FOR ACH KÄHLER MANIFOLDS}

Let $N$ be a complex manifold. We consider a domain $M \subset N$ such that $\bar{M}$ is compact with a smooth boundary $\partial M$ which is strongly pseudoconvex, i.e., there exists a smooth function $\rho$ on $\bar{M}$ such that

(1) $\rho>0$ on $M$,

(2) $\rho=0, d \rho \neq 0$ on $\partial M$,

(3) the Levi form $-\sqrt{-1} \partial \bar{\partial} \rho$ is positive near $\partial M$.

Definition 3.1. A Kähler metric $\omega$ on $M$ is called ACH (asymptotically complex hyperbolic) if near $\partial M$ we have $\omega=-2 \sqrt{-1} \partial \bar{\partial} \log \rho+\Theta$ for some defining function $\rho$, where $\Theta$ extends to $\partial M$ smoothly.

There are many examples of ACH Kähler manifolds: a strongly pseudoconvex domain $\Omega \subset \mathbb{C}^{n}$ with the Bergmann metric or the Kähler -Einstein metric constructed by Cheng and Yau [CY]. The Calabi example discussed in Section 2 is an ACH Kähler manifold. 
According to Klembeck [Kle, the curvature tensor of an $\mathrm{ACH}$ Kähler metric satisfies

$$
R_{i \bar{j} k \bar{l}}-\frac{1}{2}\left(g_{i \bar{j}} g_{k \bar{l}}+g_{i \bar{l}} g_{k \bar{j}}\right) \rightarrow 0, \quad \text { as } z \rightarrow \partial M ;
$$

i.e., it approaches the curvature tensor of constant holomorphic sectional curvature -1 , hence the name ACH (asymptotically complex hyperbolic).

One can study $L^{2}$-harmonic forms on an ACH Kähler manifold $M$ of complex dimension $m$ in the same way as Mazzeo $\mathrm{Ma}$ ] did on a conformally compact Riemannian manifold. The main point is that an $L^{2}$-harmonic form has a good asymptotic expansion and the space of $L^{2}$-harmonic $p$-forms $\mathcal{H}^{p}(M)$ is isomorphic to the relative cohomology $H^{p}(M, \partial M)$ for $p<m$ and to the absolute cohomology $H^{p}(M)$ for $p>m$. The analysis is contained in Epstein, Melrose and Mendoza EMM where the more general $\Theta$-metric is considered. The indicial components for eigenforms are computed in Epstein and Melrose [EM].

By this analytic theory, one can easily establish the following.

Theorem 3.2. Let $M$ be an ACH Kähler manifold. Then $\mathcal{H}^{1}(M)=0$.

Proof. Since $M$ is Kähler, we have the decomposition $\mathcal{H}^{1}(M)=\mathcal{H}^{1,0}(M) \oplus$ $\mathcal{H}^{0,1}(M)$, and moreover the two factors are isomorphic by conjugation. Therefore, it suffices to prove that there is no nonzero $L^{2}$-holomorphic 1-form. Suppose $\theta$ is such a form; then it vanishes on the boundary. By the strong maximum principle, it must be identically zero.

Remark 3.3. By the same proof, $\mathcal{H}^{1}(M)=0$ if we only assume that $M$ has an asymptotically complex hyperbolic end. But in general $\mathcal{H}^{1}(M)$ is not isomorphic to $H^{1}(M)$.

As $\mathcal{H}^{1}(M)=H^{1}(M, \partial M)$, we have the following by Lefschetz duality (see W1] for the analogous result in the conformally compact case).

Corollary 3.4. Let $M$ be an ACH Kähler manifold of complex dimension $m$. Then $H_{2 m-1}(M)=0$. In particular $\partial M$ is connected.

It should be emphasized that there is no curvature assumption in the above theorem, in contrast to the conformally compact case where the vanishing of $H^{1}(M)$ is true only under extra assumptions, e.g., the Einstein condition and some restriction on the conformal infinity. But if we look at the next cohomology group there is indeed a similarity.

Lemma 3.5. Let $\xi$ be a real harmonic (i.e. closed and coclosed) (1,1)-form on an n-dimensional Kähler manifold $M$. Then

$$
|\nabla \xi|^{2} \geq\left.(1+1 / n)|\nabla| \xi\right|^{2} .
$$

Moreover equality holds iff there exists a unit $(1,0)$-form $\theta$ such that

$$
\begin{aligned}
\xi & =\lambda \omega-n \sqrt{-1} \theta \wedge \bar{\theta}, \\
\nabla_{X_{0}} \xi & =t \xi, \\
\nabla_{X_{k}} \xi & \left.=t \theta \wedge X_{k}\right\lrcorner \xi,
\end{aligned}
$$

where $\left\{X_{0}, X_{1}, \ldots, X_{n-1}\right\}$ is a unitary frame for $T^{1,0} M$ with $X_{0}$ dual to $\theta$. 
Proof. We do the calculation near a point $p \in M$. In local coordinates we write $\omega=\sqrt{-1} g_{i \bar{j}} d z^{i} \wedge d \bar{z}^{j}$ and $\xi=\sqrt{-1} a_{i \bar{j}} d z^{i} \wedge d \bar{z}^{j}$. We can assume

$$
g_{i \bar{j}}(p)=\delta_{i j}, \quad d g_{i \bar{j}}(p)=0, \quad a_{i \bar{j}}(p)=\lambda_{i} \delta_{i j} .
$$

Since $\xi$ is harmonic, $\Lambda \xi=g^{i \bar{j}} a_{i \bar{j}}$ is constant. Hence

$$
\sum_{j} \frac{\partial a_{j \bar{j}}}{\partial z^{k}}(p)=0
$$

Since $\xi$ is $\partial$-closed, we have

$$
\frac{\partial a_{i \bar{j}}}{\partial z^{k}}=\frac{\partial a_{k \bar{j}}}{\partial z^{i}}
$$

We compute at $p$,

$$
\begin{aligned}
\left.4|\xi|^{2}|\nabla| \xi\right|^{2} & =\left.\left.|\nabla| \xi\right|^{2}\right|^{2} \\
& =2 \sum_{j} \frac{\partial|\xi|^{2}}{\partial z^{j}} \frac{\partial|\xi|^{2}}{\partial \bar{z}^{j}} \\
& =8 \sum_{j}\left|\sum_{i} \lambda_{i} \frac{\partial a_{i \bar{i}}}{\partial z^{j}}\right|^{2} \\
& \leq 8 \sum_{i} \lambda_{i}^{2} \sum_{i j}\left|\frac{\partial a_{i \bar{i}}}{\partial z^{j}}\right|^{2} \\
& =8|\xi|^{2} \sum_{i j}\left|\frac{\partial a_{i \bar{i}}}{\partial z^{j}}\right|^{2} .
\end{aligned}
$$

Hence

$$
\left.|\nabla| \xi\right|^{2} \leq 2 \sum_{i j}\left|\frac{\partial a_{i \bar{i}}}{\partial z^{j}}\right|^{2} .
$$

On the other hand, using (3.4),

$$
\begin{aligned}
|\nabla \xi|^{2} & =2 \sum_{i j k}\left|\frac{\partial a_{i \bar{j}}}{\partial z^{k}}\right|^{2} \\
& =2 \sum_{i \neq j, k}\left|\frac{\partial a_{i \bar{j}}}{\partial z^{k}}\right|^{2}+2 \sum_{i k}\left|\frac{\partial a_{i \bar{i}}}{\partial z^{k}}\right|^{2} \\
& =2 \sum_{i \neq j, k}\left|\frac{\partial a_{k \bar{j}}}{\partial z^{i}}\right|^{2}+2 \sum_{i j}\left|\frac{\partial a_{i \bar{i}}}{\partial z^{j}}\right|^{2} \\
& \geq 2 \sum_{i \neq j}\left|\frac{\partial a_{j \bar{j}}}{\partial z^{i}}\right|^{2}+2 \sum_{i j}\left|\frac{\partial a_{i \bar{i}}}{\partial z^{j}}\right|^{2} .
\end{aligned}
$$

By (3.3),

$$
\begin{aligned}
\sum_{i \neq j}\left|\frac{\partial a_{j \bar{j}}}{\partial z^{i}}\right|^{2} & =\sum_{i j}\left|\frac{\partial a_{j \bar{j}}}{\partial z^{i}}\right|^{2}-\sum_{i}\left|\sum_{j(j \neq i)} \frac{\partial a_{j \bar{j}}}{\partial z^{i}}\right|^{2} \\
& \geq \sum_{i j}\left|\frac{\partial a_{j \bar{j}}}{\partial z^{i}}\right|^{2}-(n-1) \sum_{i \neq j}\left|\frac{\partial a_{j \bar{j}}}{\partial z^{i}}\right|^{2}
\end{aligned}
$$


Hence $\sum_{i \neq j}\left|\frac{\partial a_{j \bar{j}}}{\partial z^{i}}\right|^{2} \geq \frac{1}{n} \sum_{i j}\left|\frac{\partial a_{j \bar{j}}}{\partial z^{i}}\right|^{2}$. Going back to the previous calculation, we obtain

$$
|\nabla \xi|^{2} \geq 2(1+1 / n) \sum_{i j}\left|\frac{\partial a_{i \bar{i}}}{\partial z^{j}}\right|^{2}
$$

Combining this inequality with (3.5), we obtain

$$
|\nabla \xi|^{2} \geq\left.(1+1 / n)|\nabla| \xi\right|^{2}
$$

From the proof we can see when the equality holds. For (3.5) to be an equality we must have

$$
\frac{\partial a_{i \bar{i}}}{\partial z^{j}}=t_{j} \lambda_{i}
$$

for some number $t_{j}$. We also have

$$
\frac{\partial a_{k \bar{j}}}{\partial z^{i}}=0, \quad \text { if } k \neq j, i \neq j
$$

and

$$
\frac{\partial a_{j \bar{j}}}{\partial z^{i}}=s_{i} \text { for } j \neq i
$$

Then it is easy to see that only one of the $t_{i}$ 's, say $t_{1}$, is nonzero and

$$
\lambda_{2}=\lambda_{3}=\ldots=\lambda_{n}=\lambda, \quad \lambda_{1}=-(n-1) \lambda,
$$

and all $\frac{\partial a_{i \bar{j}}}{\partial z^{k}}=0$ except

$$
\frac{\partial a_{j \bar{j}}}{\partial z^{1}}=\frac{\partial a_{1 \bar{j}}}{\partial z^{j}}=t_{1} \lambda_{j}
$$

In summary, the equality holds at $p$ iff in normal coordinates we diagonalize $\xi$ such that

$$
\begin{aligned}
& \xi=\sqrt{-1}\left(-(n-1) \lambda d z^{1} \wedge d \bar{z}^{1}+\sum_{j \geq 2} \lambda d z^{j} \wedge d \bar{z}^{j}\right), \\
& \nabla_{\frac{\partial}{\partial z^{1}}} \xi=t \xi \\
& \left.\nabla_{\frac{\partial}{\partial z^{k}}} \xi=t d z^{1} \wedge \frac{\partial}{\partial z^{k}}\right\lrcorner \xi \quad(k \geq 2) .
\end{aligned}
$$

Remark 3.6. The lemma, without the characterization of the equality case, was used by Li and Yau $[\mathrm{LY}]$ in the study of asymptotically flat Kähler manifolds.

Theorem 3.7. Let $M$ be a complete ACH Kähler manifold of complex dimension $n+1$ with bisectional curvatures $K_{\mathbb{C}} \geq-1 / 2$.

(1) If $\lambda_{0}(g)>n$, then $\mathcal{H}^{2}(M)=0$.

(2) If $\lambda_{0}(g)=n$ and $\mathcal{H}^{2}(M) \neq 0$, then $M$ is isometric to the Calabi example discussed in Section 2.

Remark 3.8. Recall that the bisectional curvature is defined as $-R(u, \bar{u}, v, \bar{v})$, where $u$ and $v$ are unitary $(1,0)$ vectors. If we write $u=(X-\sqrt{-1} J X) / \sqrt{2}$ and $v=$ $(Y-\sqrt{-1} J Y) / \sqrt{2}$, where $X$ and $Y$ are unit vectors and $X \perp Y, X \perp J Y$, then

$$
-R(u, \bar{u}, v, \bar{v})=R(X, Y, X, Y)+R(X, J Y, X, J Y) .
$$


By the assumption that the bisectional curvature $\geq-1 / 2$ we mean that

$$
-R(u, \bar{u}, v, \bar{v}) \geq-\frac{1}{2}(1+\langle u, \bar{v}\rangle) .
$$

Proof. We have the Hodge decomposition $\mathcal{H}^{2}(M)=\mathcal{H}^{2,0}(M) \oplus \mathcal{H}^{1,1}(M) \oplus \mathcal{H}^{0,2}(M)$. The same argument used in the proof of Theorem 3.2 shows that both $\mathcal{H}^{2,0}(M)$ and $\mathcal{H}^{0,2}(M)$ vanish. Therefore we only need to study $\mathcal{H}^{1,1}(M)$. Suppose $\phi$ is a real $L^{2}$-harmonic $(1,1)$-form. We need the following Bochner-type formula due to Bishop and Goldberg $[\mathrm{BG}]$ (cf. Wu $[\mathrm{Wu}]$ ):

$$
\frac{1}{2} \Delta|\phi|^{2}=|\nabla \phi|^{2}-\sum_{i j} R_{i \bar{j} i j}\left(\phi_{i}-\phi_{j}\right)^{2} .
$$

Here we work with a local unitary frame of $(1,0)$-forms $\left\{\theta^{0}, \theta^{1}, \ldots, \theta^{n}\right\}$ in which $\phi=\sum_{i} \phi_{i} \theta^{i} \wedge \bar{\theta}^{i}$. Therefore

$$
\begin{aligned}
\frac{1}{2} \Delta|\phi|^{2} & \geq\left.\left(1+\frac{1}{n+1}\right)|\nabla| \phi\right|^{2}-\frac{1}{2} \sum_{i j}\left(\phi_{i}-\phi_{j}\right)^{2} \\
& =\left(1+\frac{1}{n+1}\right)|\nabla| \phi \|^{2}-(n+1)|\phi|^{2}
\end{aligned}
$$

where in the last step we used the fact that $\sum \phi_{i}=0$ and $|\phi|^{2}=\sum \phi_{i}^{2}$. This can be rewritten as

$$
|\phi| \Delta|\phi| \geq\left.\frac{1}{n+1}|\nabla| \phi\right|^{2}-(n+1)|\phi|^{2} .
$$

Let $u=|\phi|^{\frac{n}{n+1}}$. We compute, using (3.12),

$$
\begin{aligned}
\Delta u & =\frac{n}{n+1}|\phi|^{-\frac{1}{n+1}} \Delta|\phi|-\frac{n}{(n+1)^{2}}|\phi|^{-\frac{1}{n+1}-1}|\nabla| \phi||^{2} \\
& =\frac{n}{n+1}|\phi|^{-\frac{1}{n+1}-1}\left(|\phi| \Delta|\phi|-\left.\frac{1}{n+1}|\nabla| \phi\right|^{2}\right) \\
& \geq-n|\phi|^{\frac{n}{n+1}}
\end{aligned}
$$

i.e., we have

$$
\Delta u \geq-n u \text {. }
$$

By the asymptotic expansion for the $L^{2}$-harmonic form $\phi$, one can show that $u \in L^{2}$ (see W2 for an analogous discussion). The above equation implies

$$
\int_{M}|\nabla u|^{2} \leq n \int_{M} u^{2}
$$

Then $\lambda_{0}(g) \leq n$. This proves the first part. We will prove the second part in the next section.

\section{THE BORDERLINE CASE}

In the borderline case, all the above inequalities must be equalities. In particular we have

$$
\Delta u=-n u
$$


and hence $u>0$ by the Harnack inequality. By Lemma 3.5 and the remark following it, there exists a unit $(1,0)$-form $\theta$ such that for any unitary frame of $(1,0)$-forms $\left\{\theta^{0}=\theta, \theta^{1}, \ldots, \theta^{n}\right\}$ and its dual frame $\left\{X_{0}=X, \ldots, X_{n}\right\}$,

$$
\begin{aligned}
& \phi=\sqrt{-1}\left(-n \lambda \theta^{0} \wedge \bar{\theta}^{0}+\sum_{j \geq 2} \lambda \theta \wedge \bar{\theta}^{j}\right)=\lambda \omega-(n+1) \lambda \sqrt{-1} \theta \wedge \bar{\theta} \\
& \nabla_{X_{0}} \phi=t \phi \\
& \left.\nabla_{X_{k}} \phi=t \theta \wedge X_{k}\right\lrcorner \phi=t \lambda \sqrt{-1} \theta \wedge \bar{\theta}^{k} \quad(k \geq 1) .
\end{aligned}
$$

Moreover

$$
R_{0 \overline{0} i \bar{i}}=\frac{1}{2}
$$

By (4.2) we have $|\phi|^{2}=n(n+1) \lambda^{2}$. Without loss of generality we assume $\lambda>0$. We compute, using (4.2),

$$
\nabla_{X_{i}} \phi=X_{i}(\log \lambda) \phi-(n+1) \lambda \sqrt{-1}\left(\nabla_{X_{i}} \theta \wedge \bar{\theta}+\theta \wedge \nabla_{X_{i}} \bar{\theta}\right) .
$$

Comparing with (4.3) yields

$$
\begin{aligned}
X(\log \lambda) & =t, \\
\nabla_{X} \theta & =a \theta, \\
\nabla_{X} \bar{\theta} & =-a \bar{\theta},
\end{aligned}
$$

and comparing with (4.4) yields for $k \geq 1$,

$$
\begin{aligned}
X_{k} \lambda & =0, \\
\nabla_{X_{k}} \theta & =b_{k} \theta, \\
\nabla_{X_{k}} \bar{\theta} & =-b_{k} \bar{\theta}-\frac{t}{n+1} \bar{\theta}^{k} .
\end{aligned}
$$

As $\omega$ is parallel, using (4.7) and (4.8) it is easy to derive

$$
0=\nabla_{X} \omega=\sqrt{-1} \sum_{k \geq 1}\left(\nabla_{X} \theta^{k} \wedge \bar{\theta}^{k}+\theta^{k} \wedge \nabla_{X} \bar{\theta}^{k}\right) .
$$

This shows that $\nabla_{X} \theta^{k}$ does not contain $\theta$ and $\nabla_{X} \bar{\theta}^{k}$ does not contain $\bar{\theta}$ for any $k \geq 1$, i.e.,

$$
\nabla_{X} \theta^{k}(X)=0, \quad \nabla_{X} \bar{\theta}^{k}(\bar{X})=0 .
$$

Similarly we have for $k \geq 1$,

$$
0=\nabla_{X_{k}} \omega=\sqrt{-1}\left(-\frac{t}{n+1} \theta \wedge \bar{\theta}^{k}+\sum_{l \geq 1} \nabla_{X_{k}} \theta^{l} \wedge \bar{\theta}^{l}+\theta^{l} \wedge \nabla_{X_{k}} \bar{\theta}^{l}\right) .
$$

This implies that

$$
\nabla_{X_{k}} \theta^{l}(X)=\frac{t}{n+1} \delta_{k l}, \quad \nabla_{X_{k}} \bar{\theta}^{l}(\bar{X})=0 .
$$

Dualizing (4.10), (4.11) and (4.13) gives

$$
\nabla_{X_{k}} X=-b_{k} X-\frac{t}{n+1} X_{k}, \quad \nabla_{X_{k}} \bar{X}=b_{k} \bar{X} .
$$


The choice of $\theta$ is not unique as it can be multiplied by a phase $e^{i \phi}$. We now remove this ambiguity by making $t$ a positive real function. Write $X=\frac{\nu-\sqrt{-1} \xi}{\sqrt{2}}$ with $\xi=J \nu$. Then by (4.6) and (4.9) it is clear that $\left\{\xi, X_{1}, \ldots, X_{n}\right\}$ span the tangent space of $\Sigma_{\lambda}$ while $\nu=\nabla \lambda /|\nabla \lambda|$ and $t=\frac{|\nabla \lambda|}{\sqrt{2} \lambda}$. We compute, using (4.14),

$$
\begin{aligned}
{\left[\xi, X_{k}\right] } & =\frac{\sqrt{-1}}{\sqrt{2}}\left[X-\bar{X}, X_{k}\right] \\
& =\frac{\sqrt{-1}}{\sqrt{2}}\left(\nabla_{X_{k}}(X-\bar{X})-\nabla_{X-\bar{X}} X_{k}\right) \\
& =\frac{\sqrt{-1}}{\sqrt{2}}\left(-\sqrt{2} b_{k} \nu-\frac{t}{n+1} X_{k}-\nabla_{X} X_{k}+\nabla_{\bar{X}} X_{k}\right) .
\end{aligned}
$$

The last two terms $\nabla_{X} X_{k}$ and $\nabla_{\bar{X}} X_{k}$ are tangent to $\Sigma_{\lambda}$ by (4.7) and (4.8). Since $\left[\xi, X_{K}\right]$ is tangential we must have $b_{k}=0$. Therefore

$$
\nabla_{X_{k}} X=-\frac{t}{n+1} X_{k}, \quad \nabla_{X_{k}} \bar{X}=0 .
$$

Let $\Pi$ be the second fundamental form $\Sigma_{\lambda}$, i.e.,

$$
\Pi(u, v)=\left\langle\nabla_{u} \nu, v\right\rangle=\frac{1}{\sqrt{2}}\left\langle\nabla_{u}(X+\bar{X}), v\right\rangle
$$

for $u, v$ tangent to $\Sigma_{\lambda}$.

By (4.15) and (4.12) it is easy to show that

$$
\Pi\left(X_{k}, X_{l}\right)=0, \quad \Pi\left(X_{k}, \bar{X}_{l}\right)=-\frac{t}{\sqrt{2}(n+1)} \delta_{k l}, \quad \Pi\left(X_{k}, \xi\right)=0, \quad \Pi\left(\bar{X}_{k}, \xi\right)=0 .
$$

To determine $\Pi(\xi, \xi)$ we first compute

$$
\begin{aligned}
\left\langle\left[X_{k}, \bar{X}_{k}\right], X\right\rangle & =\left\langle\nabla_{X_{k}} \bar{X}_{k}-\nabla_{\bar{X}_{k}} X_{k}, X\right\rangle \\
& =-\left\langle\bar{X}_{k}, \nabla_{X_{k}} X\right\rangle+\left\langle X_{k}, \nabla_{\bar{X}_{k}} X\right\rangle \\
& =\frac{t}{n+1} .
\end{aligned}
$$

Similarly we have

Therefore

$$
\left\langle\left[X_{k}, \bar{X}_{k}\right], \bar{X}\right\rangle=-\frac{t}{n+1}
$$

$$
\left[X_{k}, \bar{X}_{k}\right]=\frac{t}{n+1}(\bar{X}-X)+T,
$$

with $T$ being a linear combination of $\left\{X_{1}, \ldots, X_{n}, \bar{X}_{1}, \ldots, \bar{X}_{n}\right\}$. We now compute

$$
\begin{aligned}
R_{0 \overline{0} k \bar{k}} & =\left\langle-\nabla_{X_{k}} \nabla_{\bar{X}_{k}} X+\nabla_{\bar{X}_{k}} \nabla_{X_{k}} X+\nabla_{\left[X_{k}, \bar{X}_{k}\right]} X, \bar{X}\right\rangle \\
& =-\frac{t}{n+1}\left\langle\nabla_{\bar{X}_{k}} X_{k}, \bar{X}\right\rangle+\frac{t}{n+1}\left\langle\nabla_{\bar{X}-X} X, \bar{X}\right\rangle \\
& =-\frac{t^{2}}{(n+1)^{2}}-\frac{\sqrt{2} t}{n+1}\left\langle\nabla_{\xi} \nu, \xi\right\rangle \\
& =-\frac{t^{2}}{(n+1)^{2}}-\frac{\sqrt{2} t}{n+1} \Pi(\xi, \xi),
\end{aligned}
$$


i.e., we have shown

$$
\Pi(\xi, \xi)=-\frac{t}{\sqrt{2}(n+1)}-\frac{n+1}{2 \sqrt{2} t} .
$$

In summary we have completely determined the second fundamental form of $\Sigma_{\lambda}$ :

$$
\left\{\begin{array}{l}
\Pi(\xi, \xi)=-\frac{t}{\sqrt{2}(n+1)}-\frac{n+1}{2 \sqrt{2} t}, \\
\Pi\left(X_{k}, \xi\right)=0, \\
\Pi\left(\bar{X}_{k}, \xi\right)=0, \\
\Pi\left(X_{k}, X_{l}\right)=0, \\
\Pi\left(X_{k}, \bar{X}_{l}\right)=-\frac{t}{\sqrt{2}(n+1)} \delta_{k l} .
\end{array}\right.
$$

Since $\nu=\nabla \lambda /|\nabla \lambda|, D^{2} \lambda=|\nabla \lambda| \Pi$ on $T \Sigma_{\lambda}$. Therefore we have

$$
\left\{\begin{array}{l}
D^{2} \lambda(\xi, \xi)=-\frac{|\nabla \lambda|^{2}}{2(n+1) \lambda}-\frac{(n+1) \lambda}{2} \\
D^{2} \lambda\left(\xi, X_{k}\right)=D^{2} \lambda\left(\xi, \bar{X}_{k}\right)=0 \\
D^{2} \lambda\left(X_{k}, \bar{X}_{l}\right)=-\frac{|\nabla \lambda|^{2}}{2(n+1) \lambda} \delta_{k l} .
\end{array}\right.
$$

Then it yields

$$
\Delta \lambda=D^{2} \lambda(\nu, \nu)-\frac{(2 n+1)|\nabla \lambda|^{2}}{2(n+1) \lambda}-\frac{(n+1) \lambda}{2} .
$$

On the other hand by (4.1) we have

$$
\Delta \lambda=\frac{|\nabla \lambda|^{2}}{(n+1) \lambda}-(n+1) \lambda .
$$

Therefore

$$
D^{2} \lambda(\nu, \nu)=\frac{(2 n+3)|\nabla \lambda|^{2}}{2(n+1) \lambda}-\frac{(n+1) \lambda}{2} .
$$

By taking the limit, we get the Hessian at a critical point of $\lambda$ :

$$
D^{2} \lambda=\left(\begin{array}{cc}
-\frac{(n+1) \lambda}{2} I_{2} & \\
& 0_{2 n \times 2 n}
\end{array}\right) .
$$

This shows that at any critical point of $\lambda$ the Hessian $D^{2} \lambda$ has constant rank 2 . Therefore $\Sigma=\{x \mid \lambda(x)=B\}$ is a nondegenerate critical submanifold of dimension $2 n$, where $B=\max \lambda$. Moreover $\lambda$ has no other critical value than $B$. The complex submanifold $\Sigma$ is actually totally geodesic. Then it is not difficult to identify $M$ as the normal line bundle over $\Sigma$ with the Calabi metric. For similar discussions, cf. W1, W2.

\section{REMARKS ON THE SPECTRUM}

Let $M$ be a complete manifold, $p$ a point in $M$. By a theorem of Manin Man, if $M$ covers a compact manifold, then $\lim _{r \rightarrow \infty} \frac{\log \operatorname{Vol}(B(p, r))}{r}$ exists and is independent of $p$. In general we can define

$$
h=\limsup _{r \rightarrow \infty} \frac{\log \operatorname{Vol}(B(p, r))}{r},
$$

which usually depends on the choice of $p$. Then we have the following simple lemma. 


\section{Lemma 5.1.}

$$
\lambda_{0}(M) \leq \frac{h^{2}}{4}
$$

To see this recall that

$$
\lambda_{0}(M)=\inf _{u \in H^{1}(M)} \frac{\int_{M}|\nabla u|^{2}}{\int_{M} u^{2}} .
$$

For any $\epsilon>0$ one can use $u(x)=e^{-\frac{h+\epsilon}{2} d(p, x)}$ as a test function to get $\lambda_{0}(M) \leq$ $\frac{(h+\epsilon)^{2}}{4}$.

Let $I$ be the isoperimetric constant on $M$ which is defined as

$$
I=\inf \frac{A(\partial \Omega)}{V(\Omega)}
$$

where the inf is taken over all compact domains $\Omega \subset M$. It is well known that $\lambda_{0} \geq I^{2} / 4$. Combined with the above lemma we have

$$
\frac{I^{2}}{4} \leq \lambda_{0} \leq \frac{h^{2}}{4}
$$

For an ACH Kähler manifold $M$ of complex dimension $n$, it is easy to see that $h=n$. Therefore $\lambda_{0} \leq n^{2} / 4$. In fact one can show that the spectrum always contains the ray $\left[n^{2} / 4, \infty\right)$. In general there may exist $L^{2}$ eigenvalues below $n^{2}$. For example, for the Calabi example, $\lambda_{0}=n-1$.

This is very similar to the case of conformally compact Einstein manifolds. But we have Lee's theorem, which tells us when there is no $L^{2}$ eigenvalue.

Theorem 5.2 ([Lee]). Let $(M, g)$ be an $(n+1)$-dimensional conformally compact Einstein manifold. If its conformal infinity has nonnegative Yamabe invariant, then $\lambda_{0}(g)=n^{2} / 4$.

One expects that for ACH Kähler manifolds there should be a similar result which rules out $L^{2}$ eigenvalues under some condition on the CR structure on the boundary. In view of Lee's theorem it is reasonable to conjecture that if the boundary has nonnegative CR Yamabe invariant, then there is no $L^{2}$ eigenvalue.

\section{ACKNOWLEDGMENTS}

The author is very grateful to Rafe Mazzeo for many helpful discussions. Without his encouragement this paper would not have been completed. The author also wants to thank the referee very much.

\section{REFERENCES}

[A] M. Anderson, Einstein metrics with prescribed conformal infinity on 4-manifolds, arXiv math.DG/0105243.

[BG] R. L. Bishop and S. I. Goldberg, On the second cohomology group of a Kaehler manifold of positive curvature, Proc. Amer. Math. Soc. 16 (1965), 119-122. MR0172221 (30:2441)

[Biq] O. Biquard, Métriques d'Einstein asymptotiquement symétriques, Astérisque (2000), no. 265, vi+109. MR1760319 (2001k:53079)

[Cal] E. Calabi, Métriques kählériennes et fibrés holomorphes, Ann. Sci. École Norm. Sup. (4) 12 (1979), no. 2, 269-294. MR0543218 (83m:32033)

[Cor] K. Corlette, Hausdorff dimensions of limit sets. I, Invent. Math. 102 (1990), no. 3, 521541. MR1074486 (91k:58067) 
[CY] S. Cheng and S. Yau, On the existence of a complete Kähler metric on noncompact complex manifolds and the regularity of Fefferman's equation, Comm. Pure Appl. Math. 33 (1980), no. 4, 507-544. MR0575736 (82f:53074)

[EM] C. L. Epstein and R. B. Melrose, Shrinking tubes and the $\bar{\partial}$-Neumann problem, unpublished.

[EMM] C. L. Epstein, R. Melrose, and G. Mendoza, Resolvent of the Laplacian on strictly pseudoconvex domains, Acta Math. 167 (1991), no. 1-2, 1-106. MR.1111745 (92i:32016)

[Kle] P. Klembeck, Kähler metrics of negative curvature, the Bergmann metric near the boundary, and the Kobayashi metric on smooth bounded strictly pseudoconvex sets, Indiana Univ. Math. J. 27 (1978), no. 2, 275-282. MR0463506 (57:3455)

[Lee] J. Lee, The Spectrum of an Asymptotically Hyperbolic Einstein Manifold, Comm. Anal. Geom. 3 (1995) 253-271. MR1362652 (96h:58176)

[LY] P. Li and S. Yau, Asymptotically flat complete Kähler manifolds, Complex geometry (Osaka, 1990), Lecture Notes in Pure and Appl. Math., vol. 143, Dekker, New York, 1993, pp. 131-144. MR.1201607 (94a:53101)

[Man] A. Manning, Topological entropy for geodesic flows, Ann. of Math. (2) 110 (1979), no. 3, 567-573. MR 0554385 (81e:58044)

[Ma] R. Mazzeo, The Hodge Theory of a Conformally Compact Metric, JDG 28 (1988) 309339. MR0961517 (89i:58005)

[W1] Xiaodong Wang, On conformally compact Einstein manifolds, Math. Res. Lett. 8 (2001), no. 5-6, 671-688. MR.1879811 (2003d:53075)

[W2] Xiaodong Wang, On the $L^{2}$-cohomology of a convex cocompact hyperbolic manifold, Duke Math. J. 115 (2002), no. 2, 311-327. MR1944573 (2003m:58048)

[WY] E. Witten and S.-T. Yau, Connectedness of the Boundary in the ADS/CFT Correspondence, Adv. Theor. Math. Phys. 3 (1999) 1635-1655. MR.1812133(2002b:53071)

[Wu] H. Wu, The Bochner technique in differential geometry, Math. Rep. 3 (1988), no. 2, i-xii and 289-538. MR1079031 (91h:58031)

Department of Mathematics, Michigan State University, East Lansing, Michigan 48824

E-mail address: xwang@math.msu.edu 